Article

\title{
The Influence of Regional Freeze-Thaw Cycles on Loess Landslides: Analysis of Strength Deterioration of Loess with Changes in Pore Structure
}

\author{
Zuyong Li *(D), Gengshe Yang and Hui Liu \\ School of Architecture and Civil Engineering, Xi'an University of Science and Technology, Xi'an 710054, China; \\ yanggs@xust.edu.cn (G.Y.); woaiwojialiu@126.com (H.L.) \\ * Correspondence: Lzy12321@126.com
}

Received: 25 September 2020; Accepted: 28 October 2020; Published: 30 October 2020

\begin{abstract}
The loess landslide in Gaoling District of Xi'an, Shaanxi in China is closely related to the seasonal freeze-thaw cycle, which is manifested by the destruction of pore structure and strength deterioration of the loess body under freeze-thaw conditions. In order to study the relationship between macro-strength damage and pore structure deterioration of saturated loess under freeze-thaw conditions and its influence on the stability of landslides, this paper explores the effect of freeze-thaw cycles on the strength of saturated undisturbed loess through triaxial compression test, and explores the micro-microstructure changes of saturated undisturbed loess through scanning electron microscopy (SEM) and nuclear magnetic resonance (NMR). This is to analyze the evolution of the pore structure and strength loss evolution of saturated loess during the freeze-thaw process, and to describe the freeze-thaw damage of saturated undisturbed loess through the change of porosity and strength deterioration. Then, the internal correlation expression between the porosity change and the strength degradation is established to realize the verification analysis of the test data based on the correlation model. The research results show that: (1) As the number of freeze-thaw cycles increases, the peak strength loss rate gradually increases, and the strength deterioration of saturated loess becomes more and more obvious. (2) The freeze-thaw cycle will lead to the development of pores and cracks in the sample, accompanied by the generation of new cracks, which will cause the deterioration of the pore structure of the sample as a whole. (3) The response of strength damage and porosity deterioration of saturated undisturbed loess is roughly similar under the freeze-thaw cycle. The change in porosity can be measured to better reflect the strength deterioration of saturated loess. Therefore, the change of pore structure of undisturbed loess under freeze-thaw cycle conditions is tested by field sampling and indoor tests to reflect the phenomenon of strength deterioration, thereby analyzing the stability of loess slopes.
\end{abstract}

Keywords: freeze-thaw damage; scanning electron microscopy; nuclear magnetic resonance; porosity; strength degradation; landslide

\section{Introduction}

Loess is characterized by looseness, porosity, and easy water seepage. It is easily eroded by flowing water to form gullies, which can easily cause subsidence and collapse [1-4]. Loess is widely distributed in Northwestern China, as shown in Figure 1. Due to the special geographical location and climatic conditions, the annual temperature difference is large about $35^{\circ} \mathrm{C}$ forming a seasonal frozen soil area with regional characteristics. The region lies in a process of freezing and melting cycles all the year round, and is sensitive to thermal disturbances in the external environment [5]. The freeze-thaw cycle is part of the main factors that cause the deterioration of the physical and 
mechanical properties of loess, which aggravates the landslide hazard problem in the loess area. In the project under construction and proposed, the yellow soil foundation and slope are prone to instability and damage under the effect of seasonal freeze-thaw cycles, which will endanger the construction and associated safety [6,7]. For example, during the occurrence of a slope failure, the freeze-thaw cycle plays a catalytic role. When frozen, the ice crystal frost expansion force destroys the soil structure. The deformation is unrecoverable during melting, resulting in a decrease in strength and spalling under the softening action of water and the catalysis of ice. The freeze-thaw cycle will cause relatively intense freeze-thaw erosion on the loess, changing the soil structure and causing uneven settlement of the foundation. Therefore, in seasonally frozen soil areas, the freeze-thaw cycle is closely related to the instability of loess. So, it is necessary to further explore the effects of freeze-thaw cycles on the physical and mechanical properties of loess.

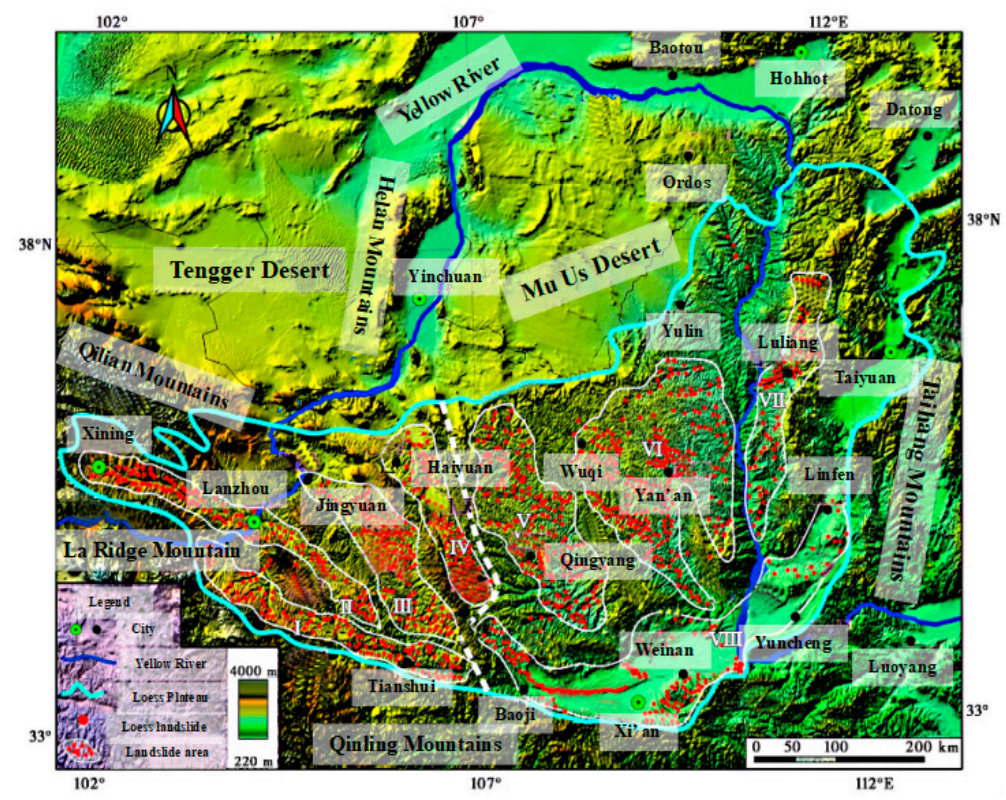

Figure 1. Spatial distribution of landslides in the Loess Plateau of the Yellow River Basin [8].

The freeze-thaw cycle is extremely destructive, which can change the internal structure of the loess and weaken its mechanical properties, leading to a decrease in the bearing capacity of the loess and causing engineering disasters. $\mathrm{Xu}$ et al. [9] studied the meso-structural characteristics of loess after freeze-thaw cycles, such as equivalent diameter, particle orientation, circularity, and pore area ratio, by comparing remolded loess with undisturbed loess. Wang et al. [10] studied the rate of change of loess volume before and after freeze-thaw by changing the blending ratio, in order to determine the reasonable ratio so that the loess can maintain its microstructure during the freeze-thaw cycle. Li et al. [11] aimed at the strength degradation and structural degradation of compacted loess under the dual effects of wet and dry and freeze-thaw. Li et al. [12] used nuclear magnetic resonance (NMR) technology to study the degradation characteristics of sandstone microstructure in freeze-thaw cycles, and used fractal theory to calculate the fractal dimension of rock pore development after different freeze-thaw cycles. Tian et al. [13] studied the freeze-thaw properties of three soil with different compositions based on nuclear magnetic resonance $(\mathrm{NMR})$ proton spin relaxation time $\left(\mathrm{T}_{2}\right)$ distribution and free induction decay (FID) measurements.

Testing the strength of freeze-thaw cycle loess is of great importance for evaluating the stability of slopes and foundations in loess areas. In order to explore the changes of mechanical properties of loess under the action of freeze-thaw cycles, it is found that the change of water content and the rate of freezing have a great influence on the strength change [9,11,14]. Yan et al. [15] studied the unconfined compressive strength and pore distribution characteristics of lime fly ash loess through a series of experiments under freeze-thaw cycles. Xu et al. [16] carried out freeze-thaw tests, direct 
shear test and scanning electron microscope on loess to study the strength characteristics of loess after freeze-thaw cycles.

At present, there are few studies on the correlation between loess strength and pore structure. This paper considers predicting the change in strength with a relatively easy to measure porosity. The saturated undisturbed loess from $\mathrm{Xi}^{\prime}$ an was subjected to triaxial compression tests under different freeze-thaw cycles to explore the law of strength degradation of samples. The pore structure of the sample after freeze-thaw cycles was observed by scanning electron microscopy and nuclear magnetic resonance. Based on the test results of strength deterioration and porosity change of saturated undisturbed loess after freeze-thaw cycles, the correlation between the two is discussed, which provides a new idea for the non-destructive analysis of the strength damage status of saturated loess by freeze-thaw.

\section{Test Scheme}

\subsection{Materials and Methods}

The soil sample used in the experiment was $Q_{3}$ undisturbed loess in Gaoling District, Xi'an, with a depth of $5-8 \mathrm{~m}$. The particle gradation curve is shown in Figure 2. Prepare a standard cylinder with a size of $39.1 \mathrm{~mm} \times 80 \mathrm{~mm}$ (diameter $\times$ height), dry and saturate the prepared sample, and seal it for later test use.

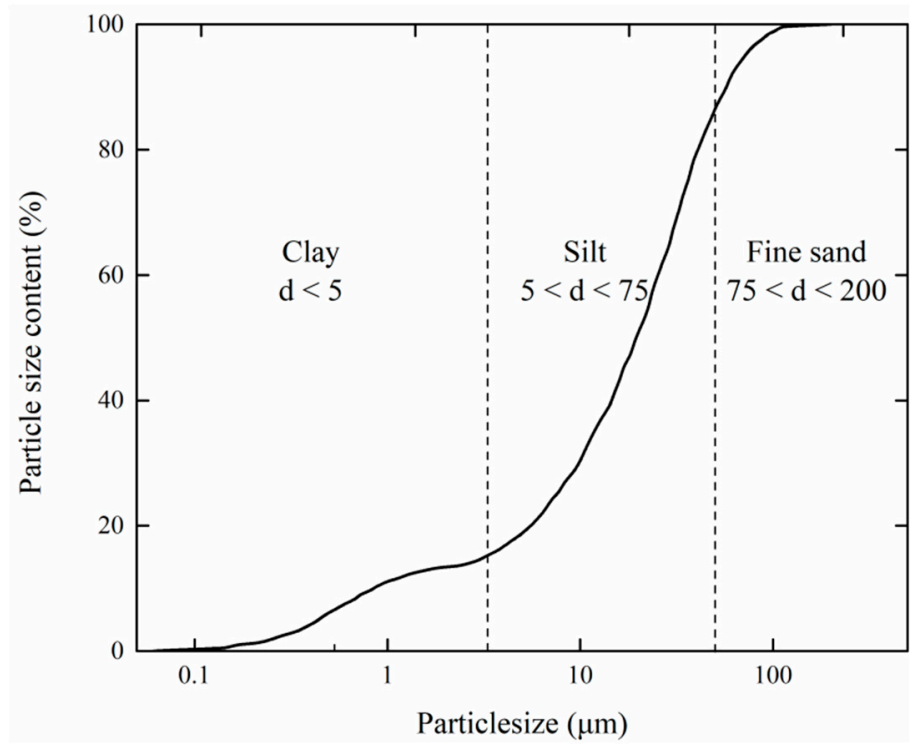

Figure 2. Soil sample particle grading curve.

\subsection{Freeze-Thaw Cycle Tests}

Frozen undisturbed loess samples were subjected to a freeze-thaw cycle test. According to the actual climate and environmental conditions in the field, the freezing temperature of the test was selected to be $-10{ }^{\circ} \mathrm{C}$ and the melting temperature was chosen to be $20^{\circ} \mathrm{C}$. Place the sample in the high and low temperature tester (type RTP-175BU, located in XUST), setting the freezing temperature to $-10{ }^{\circ} \mathrm{C}$ for $12 \mathrm{~h}$, and melt the temperature at $20^{\circ} \mathrm{C}$ for $12 \mathrm{~h}$, respectively, for $5,10,20,30$, and 50 cycle tests.

\subsection{SEM}

The sample preparation process required for scanning electron microscopy is easy. Scanning electron microscopy has the advantages of three-dimensional imaging, wide magnification of images and high resolution. Scanning electron microscopy can directly observe the fine structure of the 
uneven surface of numerous samples. Scanning electron microscopy is generally widely used in biology, physics, and chemistry [17-19]. Due to the advantages of scanning electron microscopy in observing the microstructure of samples, domestic and foreign experts have cited this technology in various fields of geotechnical engineering [20-24]. After the freeze-thaw cycle, the flat surface of the sample is selected as the scanning observation surface. A small piece of about $10 \times 10 \times 5 \mathrm{~mm}$ (length $\times$ width $\times$ height) was prepared as a scanning electron microscope sample. Scanning electron microscopy (type JSM-6460LV, located in XUST) was used to observe the microstructural changes of the loess samples under the freeze-thaw cycle.

\subsection{Nuclear Magnetic Resonance Tests}

At present, NMR technology is gradually applied to geological exploration engineering due to its magnetic field characteristics [25-27]. NMR technology can observe the internal pore structure of the porous material, the molecular motion state in the pores and the reaction process through fluid changes in the pores, and the relationship between these phenomena. Therefore, nuclear magnetic resonance technology is cited in the field of geotechnical engineering to observe the meso-structural changes of the rock and soil. After the freeze-thaw cycle, the pore size distribution of the loess samples under the freeze-thaw cycle was observed using a large-aperture nuclear magnetic resonance imaging analyzer (type MacroMR12-150H-I, located in XUST, Xi'an, China).

\subsection{Triaxial Compression Tests}

In order to study the influence of freeze-thaw cycles on the mechanical properties of loess, the TSZ-6A strain-controlled triaxial apparatus was used to carry out triaxial compression tests under different confining pressures on the samples that reached the cycle number. The stress-strain curves and corresponding mechanical parameters of samples under different freeze-thaw cycles are obtained. The changing response of the mechanical properties of Gaoling loess under the coupling effect of different confining pressures and freeze-thaw cycles was analyzed. The instrument used in the experiment is shown in Figure 3.
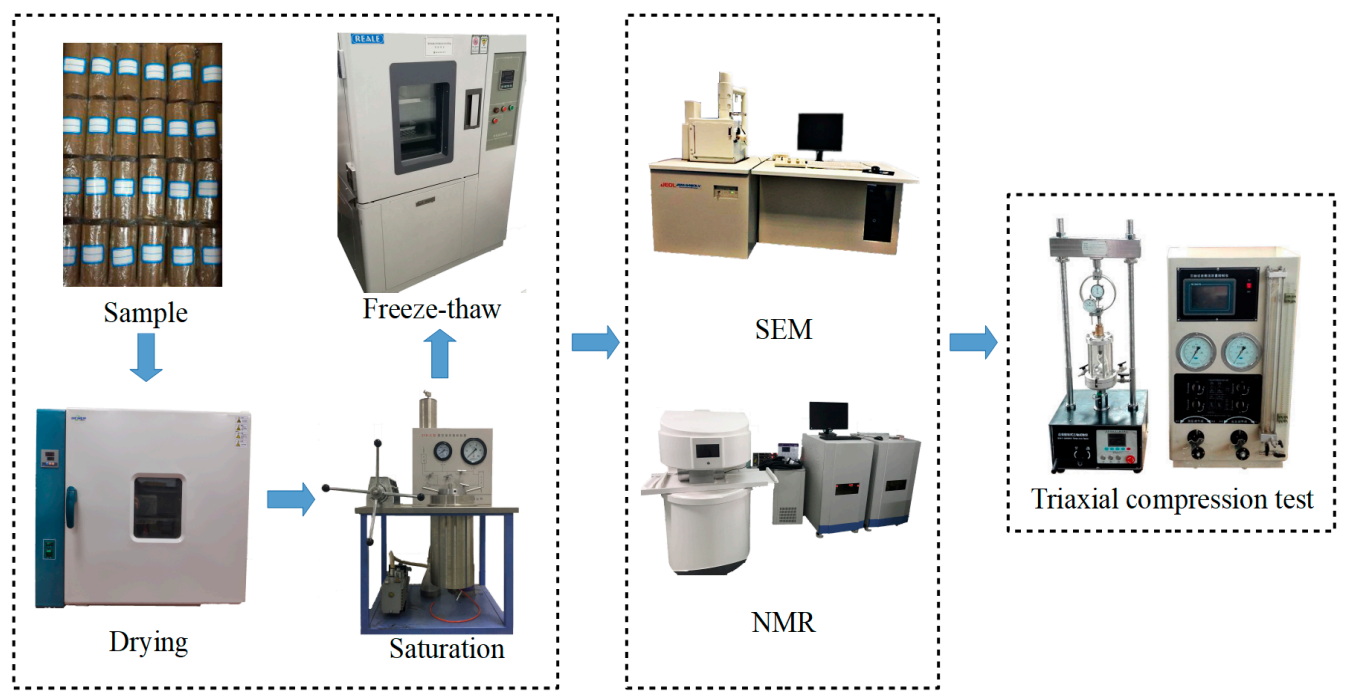

Figure 3. Test equipment.

\section{Test Results}

\subsection{SEM Results}

Scanning electron microscopy (SEM) was used to observe the microstructure changes of saturated undisturbed loess samples after freeze-thaw cycles. Select 500×, 1000×, 1500×, and 2000× 
magnifications in order from high to low magnification in five representative areas to take pictures. The microstructure image of the sample after the freeze-thaw cycle is obtained, as shown in Figure 4. The 500× magnification photo can provide more analysis objects in the same field of view. Under the condition that the soil particles are heterogeneous and anisotropic, the geometric analysis results meet the statistical requirements, avoiding the occurrence of large data errors, which is convenient for quantitative analysis. It can be seen from the results that there are pores of different sizes and shapes in the loess samples. Particle connection methods mainly include cementation, point-to-surface contact, and surface-to-surface contact.

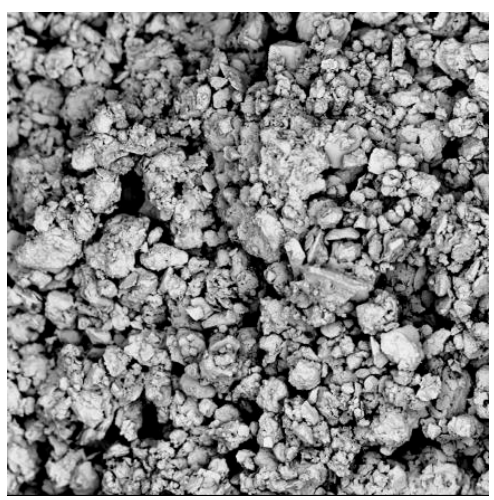

(a)

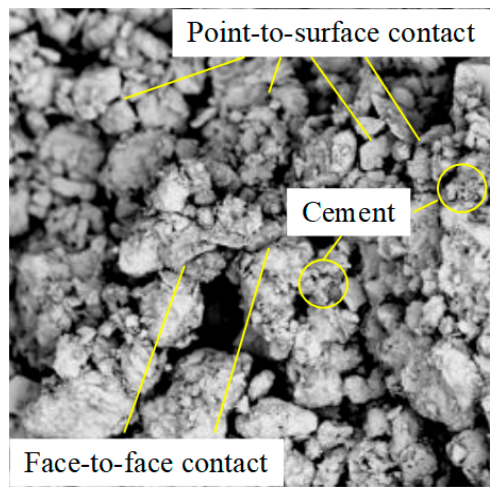

(c)

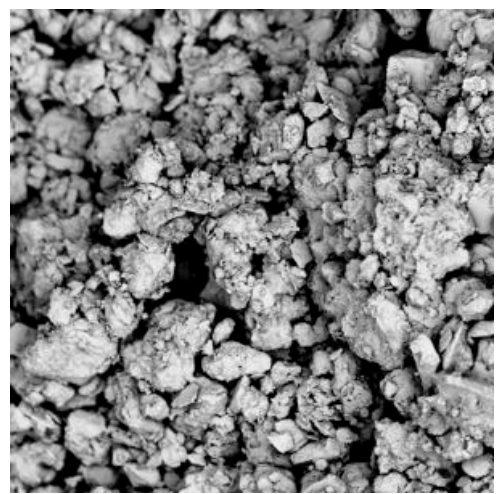

(b)

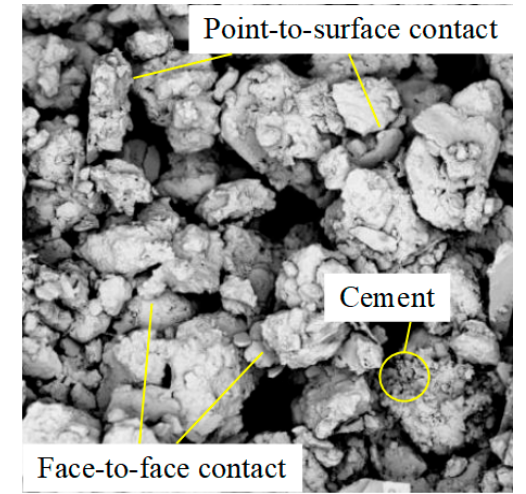

(d)

Figure 4. SEM Image. (a) 500×, (b) 1000×, (c) 1500×, (d) 2000×.

\subsection{NMR Test Results}

A nuclear magnetic resonance imaging analyzer (NMR) was used to observe the mesostructure change of the saturated undisturbed loess sample after the freeze-thaw cycle, thereby obtaining a $\mathrm{T}_{2}$ spectrum distribution curve of the sample, as shown in Figure 5 ( $T_{2}$ is the relaxation time of the pore fluid). The $\mathrm{T}_{2}$ spectral distribution showed a significant right shift after the freeze-thaw cycle. As the number of freeze-thaw cycles increases, the two peaks move upwards as a whole, and the trough between the two peaks becomes less obvious as the curve moves up. It shows that the expansion of the internal pores of the loess is in a process of dynamic change under the conditions of freeze-thaw cycles. Therefore, the micropores in the soil are gradually transitioning to larger pores as the number of freeze-thaw cycles increases. 


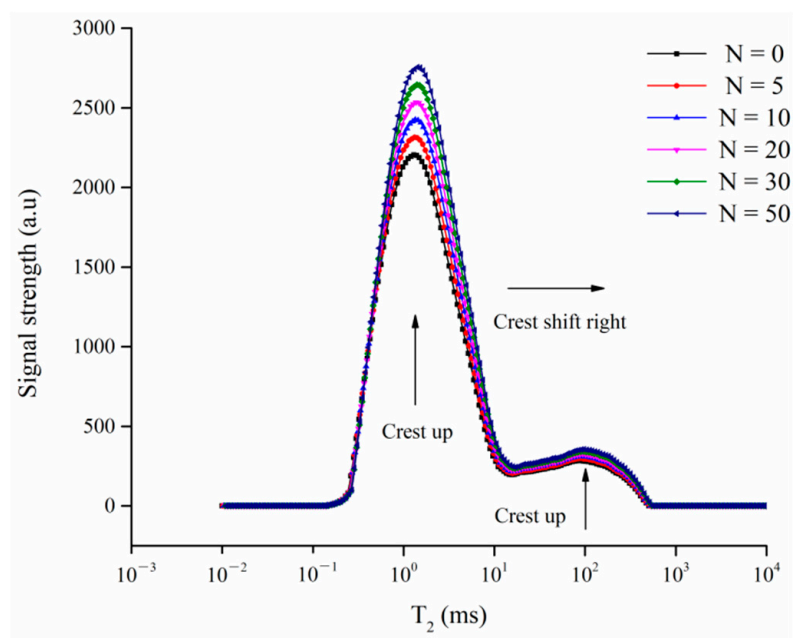

Figure 5. $\mathrm{T}_{2}$ spectral curve distribution under different freeze-thaw cycles $(\mathrm{N}$ is the number of freeze-thaw cycles).

\subsection{Triaxial Compression Test Results}

A TSZ-6A strain-controlled triaxial apparatus was used to perform a triaxial compression test on the saturated undisturbed loess processed through freeze-thaw cycles to obtain stress-strain curves under different freeze-thaw cycles, as shown in Figure 6.

(1) The freezing and thawing times of loess are divided into 6 gradients. The peak intensity decreases with increasing freeze-thaw times, under different confining pressure conditions.

(2) The greater the number of freeze-thaw cycles, the less the peak intensity degradation of the gradient compared to the previous one.

(3) As the confining pressure increases, the phenomenon of peak intensity degradation becomes less obvious.

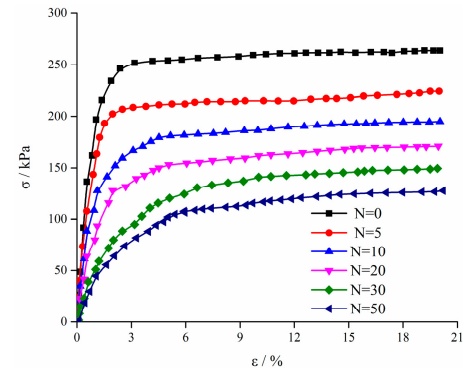

(a)

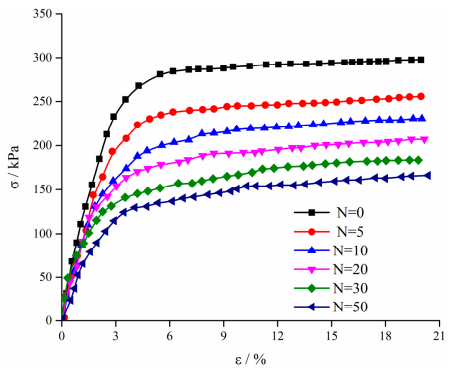

(b)

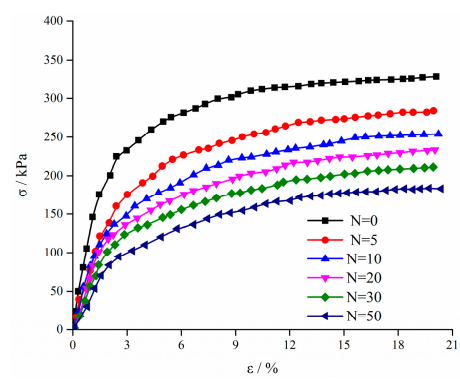

(c)

Figure 6. Stress-strain curves under different confining pressures under freeze-thaw cycles. (a) $\sigma_{3}=200 \mathrm{kPa}$, (b) $\sigma_{3}=300 \mathrm{kPa},(\mathbf{b}) \sigma_{3}=300 \mathrm{kPa},(\mathbf{c}) \sigma_{3}=400 \mathrm{kPa}$. 


\section{Discussion}

\subsection{Strength Damage}

According to the Mohr Coulomb strength criterion, combined with the results of the triaxial shear test of loess, the shear strength and corresponding mechanical parameters under different conditions are obtained, as shown in Figure 7. Figure 7 shows the relationship between the shear strength loss rate of saturated undisturbed loess and the number of freeze-thaw cycles. The shear strength degradation loss rate $\lambda$ is:

$$
\lambda=\frac{Q_{0}-Q(n)}{Q_{0}}
$$

where $Q_{0}$ is the shear strength of the unfrozen-thawed saturated undisturbed loess; $Q(n)$ is the shear strength when the number of freeze-thaw cycles is $n$ times.

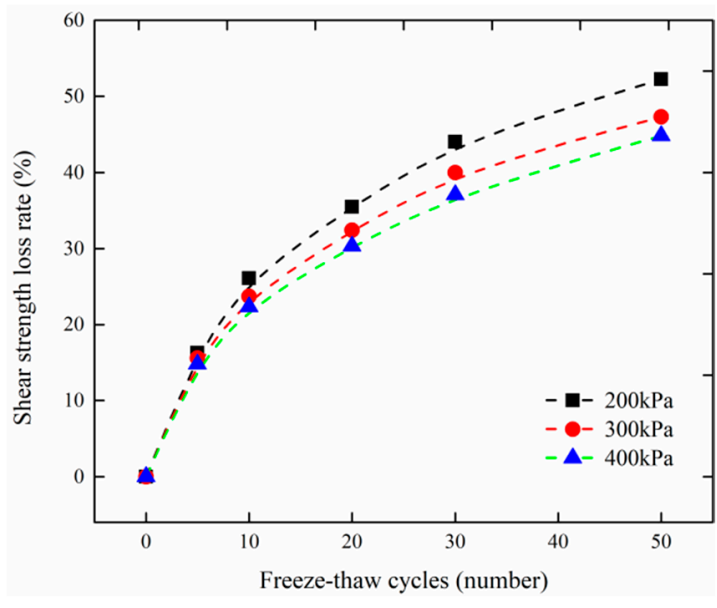

(a)

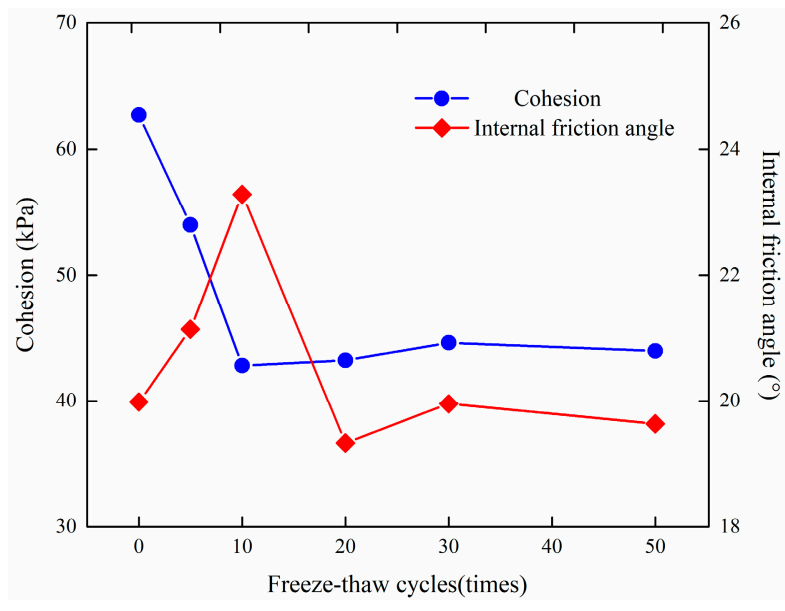

(b)

Figure 7. Strength degradation. (a) Shear strength loss rate under freeze-thaw cycles. (b) Mechanical parameters.

It can be seen from Figure 7 that as the number of freeze-thaw cycles increases, the shear strength loss rate increases gradually, and the saturated undisturbed loess strength degradation phenomenon becomes more and more obvious, and the evolution law of peak strength loss rate under different confining pressures is about the same.

It can be seen from Figure $7 \mathrm{~b}$ that as the number of freeze-thaw cycles increases, the cohesion of loess shows a tendency to decrease first, then increase slightly, and gradually stabilize and the internal friction angle showed a trend of increasing first, then decreasing and gradually stabilizing. When the freezing and thawing cycle is $0 \sim 10$ times, the cohesion of the soil sample is obviously reduced, and the internal friction angle is obviously increased. After 10 freeze-thaw cycles, the cohesion and internal friction angle of the soil sample increased slowly, and tended to be stable.

This is because the freeze-thaw cycle destroys the inherently strong cementation between the loess particles. The water in the soil generates frost heave force and migration force due to freezing and migration, which constantly weakens the bonding force between the soil particles, resulting in a decrease in cohesion. In the process of rearrangement of soil particles, the contact points between the particles are increased, resulting in an increase in the internal friction angle. As the number of freeze-thaw cycles increases, the soil particles gradually form a new stable structure. The contact point and contact mode between the particles is changed. The effect of freeze-thaw cycles on the bonding force and internal friction angle gradually decreases. Due to the formation of new structural features, the bond between the soil particles is stabilized. It reflects the resistance to freeze-thaw damage, so the cohesive force and internal friction angle change are small. Therefore, it is believed that 
when the number of freeze-thaw cycles is small, the effect of freeze-thaw cycles on cohesive force and the internal friction angle is more obvious. As the number of freeze-thaw cycles increases, its effect gradually decreases.

\subsection{SEM Test Result Analysis}

The binarization of the image is conducive to the further processing of the image, making the image simple, reducing the amount of data, and highlighting the outline of the target. The microstructure of the loess sample was obtained by scanning electron microscopy, and the SEM image (Figure 4a, $500 \times$ ) was selected for quantitative analysis. In order to obtain the characteristic parameters of the microstructure of the loess, the SEM image is binarized, as shown in Figure 8.

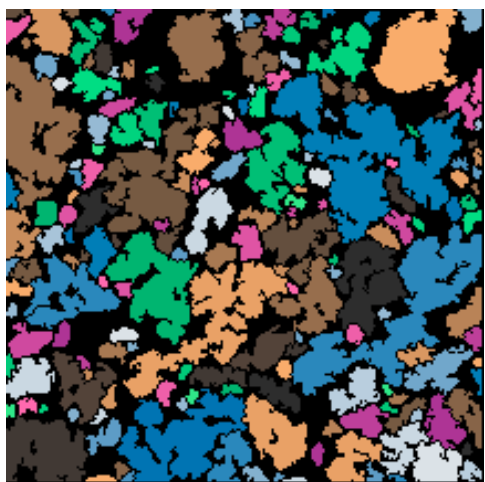

(a)

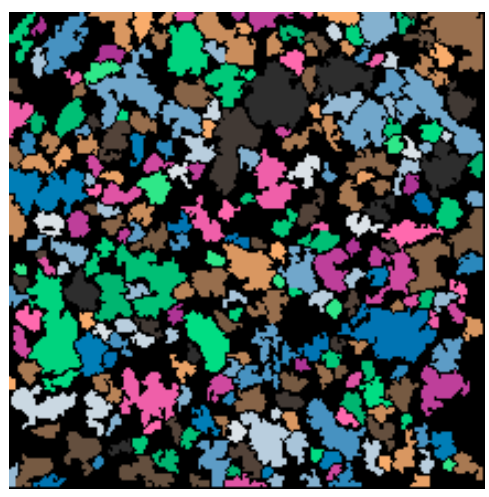

(c)

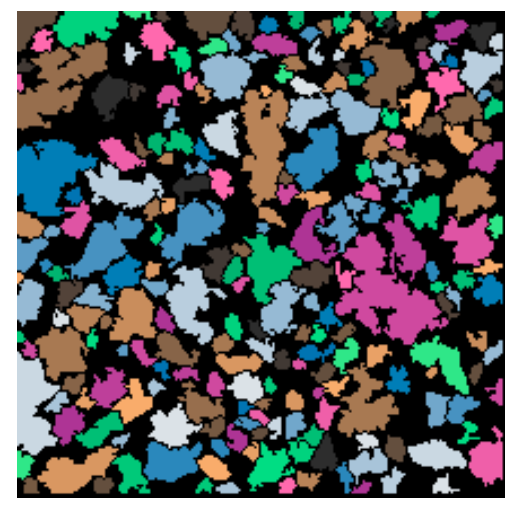

(e)

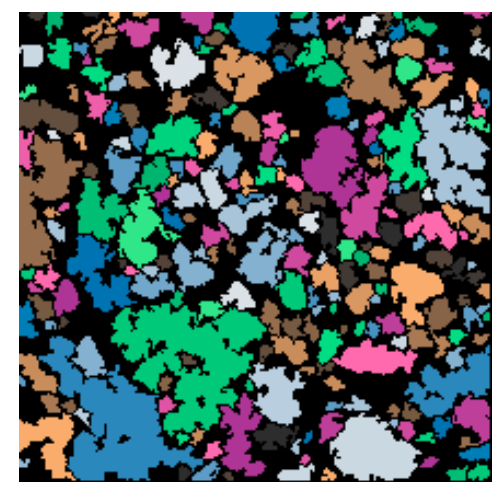

(b)

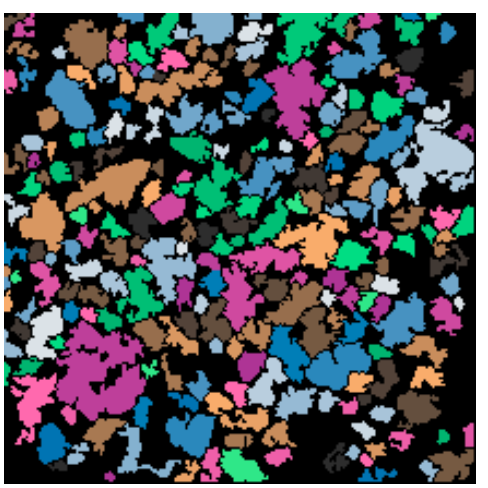

(d)

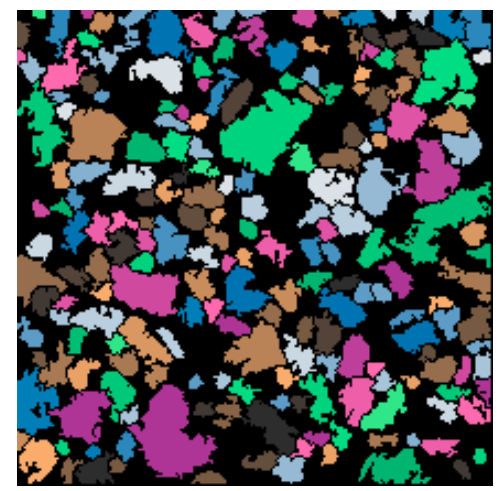

(f)

Figure 8. SEM image. (a) 0, (b) 5, (c) 10, (d) 20, (e) 30, (f) 50. 
The fractal dimension can vividly reflect the morphological characteristics, arrangement characteristics and size distribution characteristics of loess particles. In order to explore the influence of freeze-thaw times on the distribution of loess particles, the SEM image was binarized to obtain the statistical parameters of the statistical particles of saturated loess under different freeze-thaw times. Probability entropy and area probability distribution index are selected to elaborate the fractal dimension of loess.

(1) Probability entropy

Probability entropy is a structural parameter describing the arrangement of particles, which can be used to analyze the arrangement of soil particles after the freeze-thaw cycle. The calculation equation of probability entropy is

$$
H_{m}=-\sum_{i=1}^{n} \frac{m_{i}}{M} \cdot \frac{\ln \left(m_{i} / M\right)}{\ln n}
$$

where $H_{m}$ is the probability entropy, and the value range is [0,1]. The larger the value, the lower the order of the particle arrangement and the more chaotic the arrangement. Divide $0 \sim 180^{\circ}$ into $n$ zones, and the angle range of each zone. $m_{i}$ is the number of particles whose particle long axis is in the $i$-th interval. $M$ is the total number of particles.

It can be seen from Figure 9a that the probability entropy of soil particles gradually increases under the action of freeze-thaw cycles. As the number of freeze-thaw cycles increases, this is a neat method, which indicates that the orientation of the soil particles changes under the action of freeze-thaw cycles. As the number of freeze-thaw cycles increases, the order of particle arrangement gradually changes from a regular state to a chaotic state, and the direction of particle arrangement becomes worse and worse. This is because, during the freeze-thaw cycle, changes in external temperature conditions cause the pore water to undergo a phase change, forming a certain volume of ice crystal structure. Adjacent soil particles are relatively displaced by the wedge-shaped force of ice crystals. Due to the weakening of the bonding force, the bonding between the soil particles becomes weak, which provides conditions for the displacement of the soil particles. Therefore, under the action of freeze-thaw cycles, deflection and displacement occur between the soil particles, and the relative displacement between the particles changes the arrangement of the soil particles.

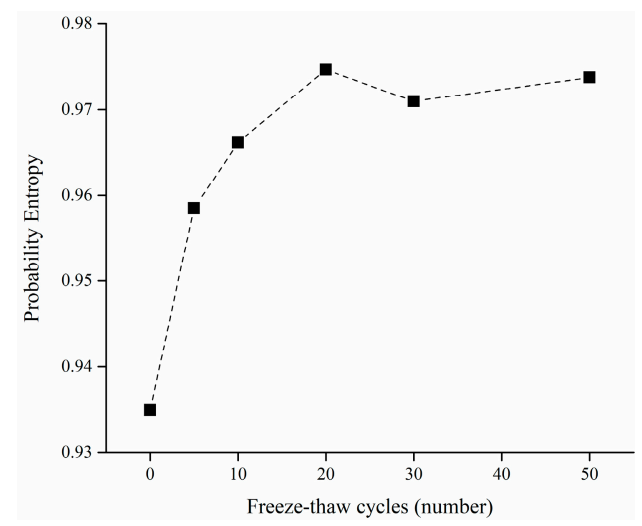

(a)

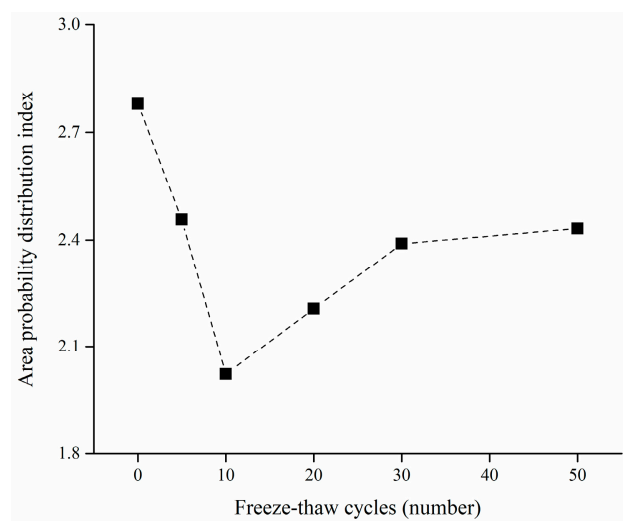

(b)

Figure 9. Fractal dimension. (a) Probability entropy, (b) Area probability distribution index.

(2) Area probability distribution index

The significance of the area probability distribution index is that it can effectively quantify the distribution of the particle area, which is defined by the probability distribution function and reflects the density of the particle area in a specific area. The probability distribution function is

$$
f(d)=a x^{-b}
$$


where $f(d)$ is the density of particles of corresponding diameter; $b$ is the probability distribution index; the value of $a$ is related to $b$. The probability distribution index describes the changing trend of the number of particles when the area of the particles changes from small to large, showing a power function relationship. When the probability distribution index is small, it indicates that there are fewer particles in small area and more particles in large area.

It can be found from Figure $9 \mathrm{~b}$ that under the action of freeze-thaw cycles, the area probability distribution index of particles decreases first and then increases, and gradually stabilizes, which indicates that with the increase of the number of freeze-thaw cycles, the number of large-area particles first increase and then decrease, and the quantity change tends to be stable. This is due to the weakening of the binding force under the action of freeze-thaw cycles, which causes the separation of the base-type aggregates. The process of debris detachment makes the space volume of the basal-type aggregates increase continuously, and the surface area gradually increases. Until the debris is separated, the surface area of the base-type aggregate decreases. Moreover, the structural characteristics of the aggregates have changed to form attachment-type and breccia-type aggregates.

Therefore, it can be obtained by SEM technology that when the number of freeze-thaw cycles is small, the internal pores of the saturated undisturbed loess change from larger micropores to smaller micropores. After reaching a certain number of cycles, pores and fissures develop and gradually transform into medium and large pores. Large holes appear and the soil structure is basically destroyed. Under the action of freeze-thaw cycle, the saturated undisturbed loess develops pores and cracks in the sample, and is accompanied by new cracks. The soil skeleton structure is destroyed, resulting in reduced bearing capacity. Therefore, the loess samples show the phenomenon of increased pores and decreased shear strength.

\subsection{NMR Test Result Analysis}

In the NMR test, the pore water content and its distribution curve of the sample can be obtained by inverting the $T_{2}$ spectrum distribution curve in Figure 5 . The $T_{2}$ value and pore structure in the soil satisfy the following equation:

$$
\frac{1}{T_{2}} \approx \rho_{2} \frac{S}{V}
$$

where $\rho_{2}$ is the transverse relaxation rate; $S$ and $V$ is the surface area and volume of the pores where the moisture is located.

Assuming that the pore shape is cylindrical, Equation (4) is simplified as:

$$
\frac{1}{T_{2}} \approx \rho_{2} \frac{2}{R}
$$

where $R$ is the pore radius. It can be seen from Equation (5) that the $T_{2}$ spectral value is proportional to the pore radius.

The $\mathrm{T}_{2}$ spectrum curve obtained by the NMR experiment was subjected to the inversion of the Equation (5) to obtain a pore distribution curve of saturated undisturbed loess, as shown in Figure 10. It can be seen from Figure 8 that the pore distribution of the loess mainly has two peaks, and the larger the peak value, the larger the proportion of the pore volume corresponding to the pore diameter. When NMR is used to test the water in the soil, according to the relaxation time $\left(T_{2}\right)$ of the water in different occurrence states, after inversion by Equation (5), it can be divided into 4 types of pores according to the pore size [28]. 


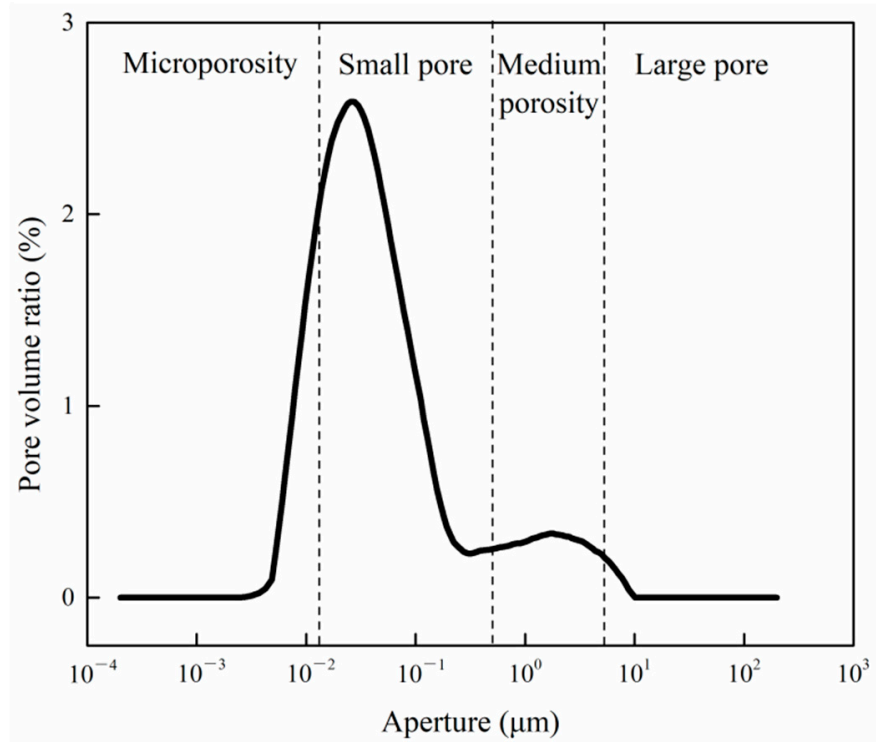

Figure 10. Pore distribution curve of saturated undisturbed loess.

It can be observed in Figure 11 that the internal structure of saturated undisturbed loess changes under the action of freeze-thaw cycles. The intergranular cementation ability is weakened. Large particles are decomposed into small particles under the action of force and migrate to nearby microcracks, resulting in a decrease in micropores and an increase in small pores. As the number of freeze-thaw cycles increases, micro-cracks and micro-voids develop new cracks. Micro and small pores are reduced, and medium and large pores are increased. The soil structure is essentially destroyed. Therefore, with the increase of the number of freeze-thaw cycles, the microporosity and small pores in the soil show an overall decreasing trend, and the medium and large pores show an overall increasing trend.

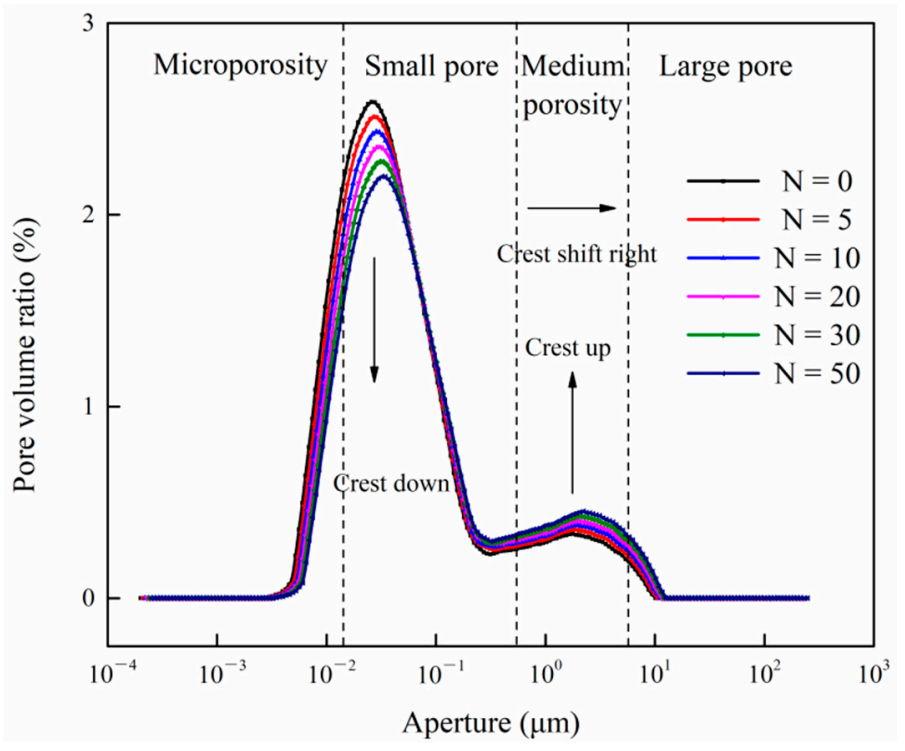

Figure 11. Pore distribution map of loess under freeze-thaw cycles.

As shown in Figure 12, micropore volume accounts for $28.35 \%$ to $37.93 \%$. The small pore volume accounts for $40.06 \%$ to $52.11 \%$. Medium pore volume accounts for $7.93 \%$ to $17.48 \%$. The large pore volume accounts for $2.04 \%$ to $10.7 \%$. The soil has the largest number of small pores, followed by micropores, mesopores, and macropores. It can be found from Figure 12 that as the number of freeze-thaw cycles increases, the variation of pore volume of different types is different, indicating that the soil has different freeze-thaw damage rates at different stages. 
(1) The freeze-thaw cycle is 0 to 10 times, and the pore volume of each type varies greatly, which indicates that the soil mesostructure has a higher degree of freeze-thaw damage and a faster damage rate.

(2) When the number of freeze-thaw cycles is 10 30, the change of pore volume of each type is small, which shows that the degree of freeze-thaw damage of the mesostructure of the soil body is reduced and the damage rate is reduced. This is because the soil forms new pore structure characteristics, which changes the degree of influence of the freeze-thaw cycle on the microstructure of the soil. After the number of freeze-thaw cycles reached 50 times, there was almost no change in the pore volume of each type. This was because the soil was basically destroyed and the pore structure was stabilized to a certain extent.

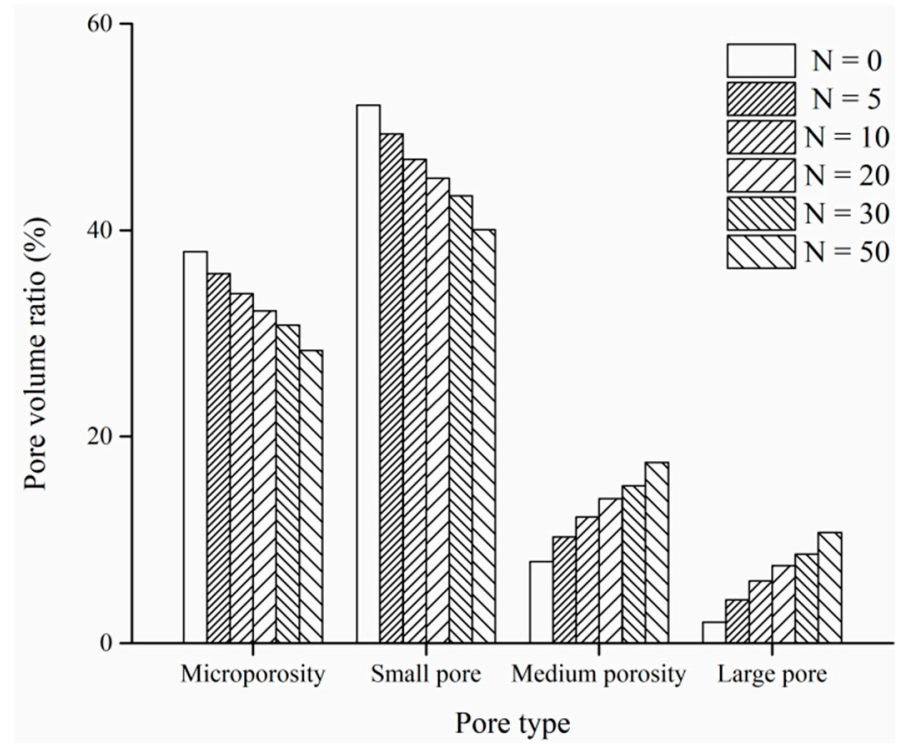

Figure 12. Pore volume distribution.

\subsection{Correlation Between Strength and Pore Structure}

\subsubsection{Changes in Porosity}

The porosity of saturated undisturbed loess under different cycle times is obtained by saturation net weight method. Figure 13 shows the relationship between the change in porosity of saturated undisturbed loess and the number of freeze-thaw cycles. The amount of change in porosity is:

$$
\Delta P=P(n)-P_{0}
$$

where $P(n)$ is the porosity of saturated undisturbed loess when the number of freeze-thaw cycles is $n$; $P_{0}$ is the porosity of saturated undisturbed loess during unfrozen and thaw. 


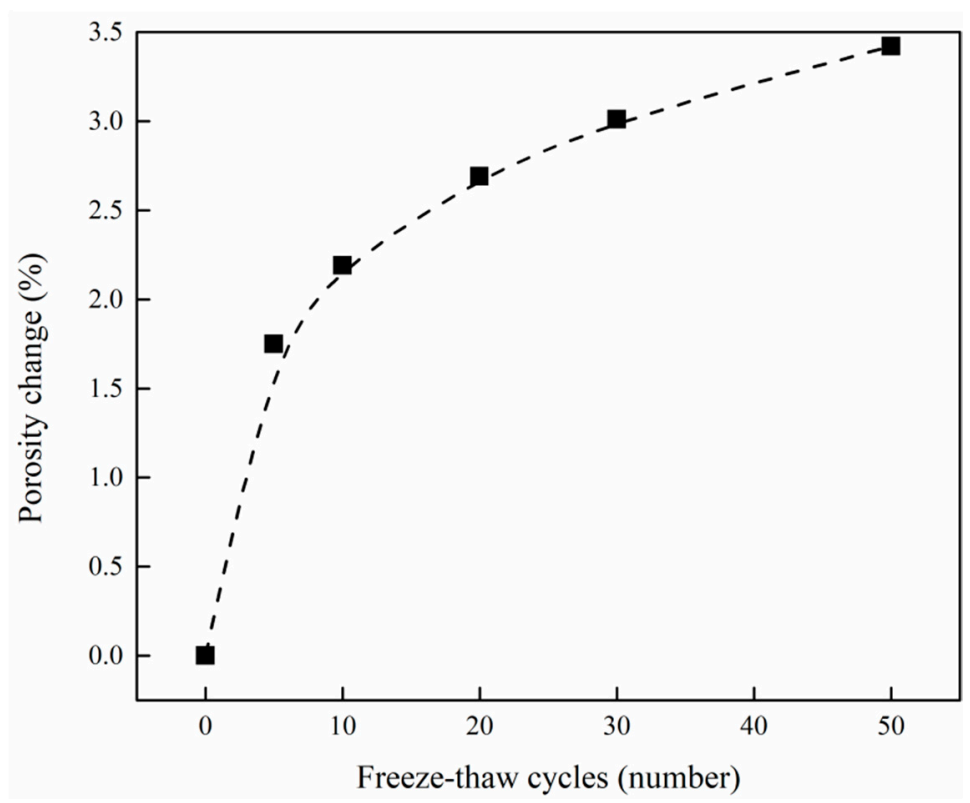

Figure 13. Relationship between freeze-thaw cycles and porosity change.

The most obvious manifestation of the damage caused by the freeze-thaw cycle is the increase of the porosity of the porous sample [29]. Therefore, the change in porosity can directly reflect the evolution of freeze-thaw damage. So, the change in porosity can be used to calculate the damage variable. According to the strain equivalence principle proposed by Lematire [30], considering the damage state of a section of the material, the damage variable $D$ can be determined according to the pore area, effective bearing area and total area of the section.

$$
D=\frac{A^{\prime}}{A}
$$

where $A^{\prime}$ is the area of the pores in a section of the material; $A$ is the total area on a section of the material.

When studying the damage mechanical properties of a section of porous media, $A^{\prime} / A$ is the damage variable on the section and the surface porosity of the section. Jia et al. [31] proposed a certain corresponding conversion relationship between surface porosity and volume porosity.

$$
D=\frac{A^{\prime}}{A}=f(P)
$$

where $P$ is the sample porosity; $f(P)$ is the conversion relationship between the surface porosity and the volume porosity.

Considering that the material is subjected to freeze-thaw cycles, the freeze-thaw damage variable $D_{\mathrm{n}}$ can be defined as:

$$
D_{\mathrm{n}}=f\left(P_{\mathrm{n}}\right)-f\left(P_{0}\right)
$$

where $D_{\mathrm{n}}$ is the damage when the number of freeze-thaw cycles is $n ; P_{\mathrm{n}}$ is the porosity when the number of freeze-thaw cycles is $n ; P_{0}$ is the initial porosity.

In order to study the relationship between surface porosity and volume porosity in detail, the parameters $k$ and the concept of feature voxels $V_{e}^{S}$ are introduced to obtain the relationship between surface porosity and volume porosity [31].

$$
f(P)=k P \quad\left(k=m V_{\mathrm{e}}^{\mathrm{S}}\right)
$$


where $m$ is a constant, $V_{e}^{S}$ is the volume of the characteristic voxel. The definition of the characteristic voxel is as follows: Take a series of voxels $V_{e}^{S}(S=1,2, \ldots)$ around any point $\mathrm{p}$ in the medium. Corresponding to each voxel, an average porosity can be obtained. As the volume of the voxel changes from small to large, the corresponding porosity fluctuations gradually decrease. When the porosity stabilizes to a certain value, the corresponding voxel is called the characteristic voxel $V_{e}$. The porosity at this time is the porosity of the medium.

Substituting Equation (10) into Equation (9) gives:

$$
D_{\mathrm{n}}=k\left(P_{\mathrm{n}}-P_{0}\right) .
$$

Since the porosity changes finitely when the material is subjected to freeze-thaw damage, that is, the volume change is not obvious, the parameter $k$ is considered to be constant.

As shown in Figure 13, the change in porosity of saturated undisturbed loess increases with the number of freeze-thaw cycles. It is assumed that the loess porosity is $P(n)$ after $\mathrm{n}$ freeze-thaw cycles, and it is a microscopic function. When the freeze-thaw cycle is $(n+\Delta n)$ times, the porosity is $P(n+\Delta n)$. From Equation (1), the amount of change in porosity from $n$ to $(n+\Delta n)$ freeze-thaw cycles is:

$$
P(n+\Delta n)-P(n)=\Delta P .
$$

Then, the relationship between the amount of porosity change and freeze-thaw damage can be obtained from Equations (11) and (12).

$$
k[P(n+\Delta n)-P(n)]=D
$$

According to the strain equivalence principle proposed by Lematire [30], the initial damage state of the material is defined as the baseline damage state. Considering that the material will deteriorate after the freeze-thaw cycle, the freeze-thaw damage variable $D_{\mathrm{n}}$ can be determined by the stress change before and after freeze-thaw cycle.

$$
D_{\mathrm{n}}=\frac{\sigma_{0}-\sigma_{\mathrm{n}}}{\sigma_{0}}
$$

As shown in Figure 7a, the shear strength loss rate of saturated undisturbed loess increases with the number of freeze-thaw cycles. The shear strength of saturated undisturbed loess after $\mathrm{n}$ freeze-thaw cycle is $Q(n)$, and it is a differentiable function. The shear strength of the loess with $(n+\Delta n)$ freeze-thaw cycles is $Q(n+\Delta n)$. From Equation (10), shear strength loss rate from $\mathrm{n}$ freeze-thaw cycles to $(n+\Delta n)$ freeze-thaw cycles is:

$$
\frac{Q(n)-Q(n+\Delta n)}{Q(n)}=\lambda
$$

Then, the relationship between the shear strength loss rate and the freeze-thaw damage can be obtained from the Equations (14) and (15).

$$
\frac{Q(n)-Q(n+\Delta n)}{Q(n)}=D
$$

The degree of strength deterioration is characterized by the change of porosity of the loess sample under the freeze-thaw cycle. Equations (13) and (16) indicate the freeze-thaw damage of the sample by the change of porosity and shear strength, respectively. Then it can be concluded:

$$
\frac{Q(n)-Q(n+\Delta n)}{Q(n)}=k[P(n+\Delta n)-P(n)] .
$$


Available from Equation (17)

$$
\frac{d Q(n)}{Q(n)}=-k d P(n)
$$

Integrate the two sides of Equation (18)

$$
\frac{Q(n)}{Q_{0}}=e^{-k\left[P(n)-P_{0}\right]} .
$$

Letting $\Delta P=P(n)-P_{0}, k$ is considered as the strength degradation factor of saturated undisturbed loess freeze-thaw cycles. Considering the influence of external conditions on the peak strength test results, the correction coefficient $\alpha$ is introduced to correct the equation, see Equation (20).

$$
\frac{Q(n)}{Q_{0}}=\alpha \cdot e^{-k \cdot \Delta P}
$$

It can be seen from Equation (20) that the relationship between the shear strength of saturated undisturbed loess and the change in porosity obeys an exponential function under the freeze-thaw cycle.

\subsubsection{Verification of Test Results}

Based on theoretical derivation, Equation (20) is used to fit the peak strength of saturated undisturbed loess and the change of porosity under the action of freeze-thaw cycles, as shown in Figure 14. The peak strength of saturated undisturbed loess has a good correlation with the change of porosity. Therefore, using the porosity change of saturated undisturbed loess before and after freezing and thawing can effectively simulate the strength deterioration law.

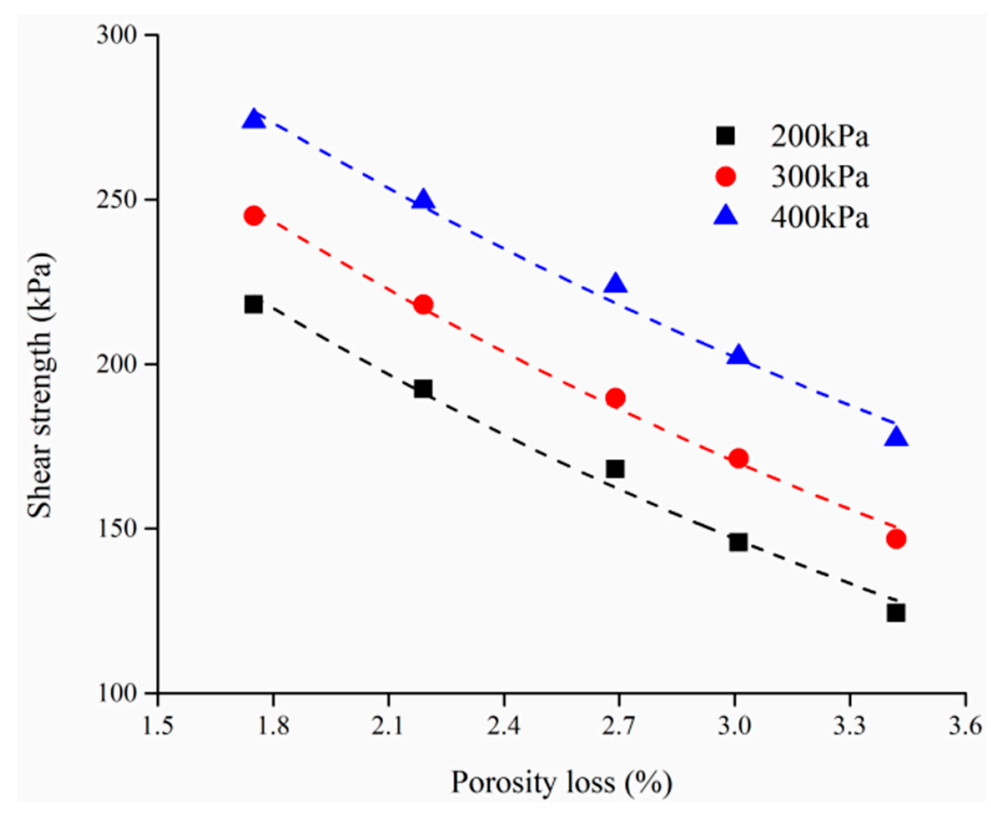

Figure 14. The relationship between shear strength and porosity change.

As the confining pressure increases, the ability of the sample to resist deformation is enhanced, and the deterioration phenomenon is weakened. As shown in Figure 15, the strength degradation factor gradually decreases as the confining pressure increases. The variation range of the correction coefficient $\alpha$ is small, and the values are not much different. It can be concluded that this test was realized under the same external influence, and the data obtained are true and reliable. 


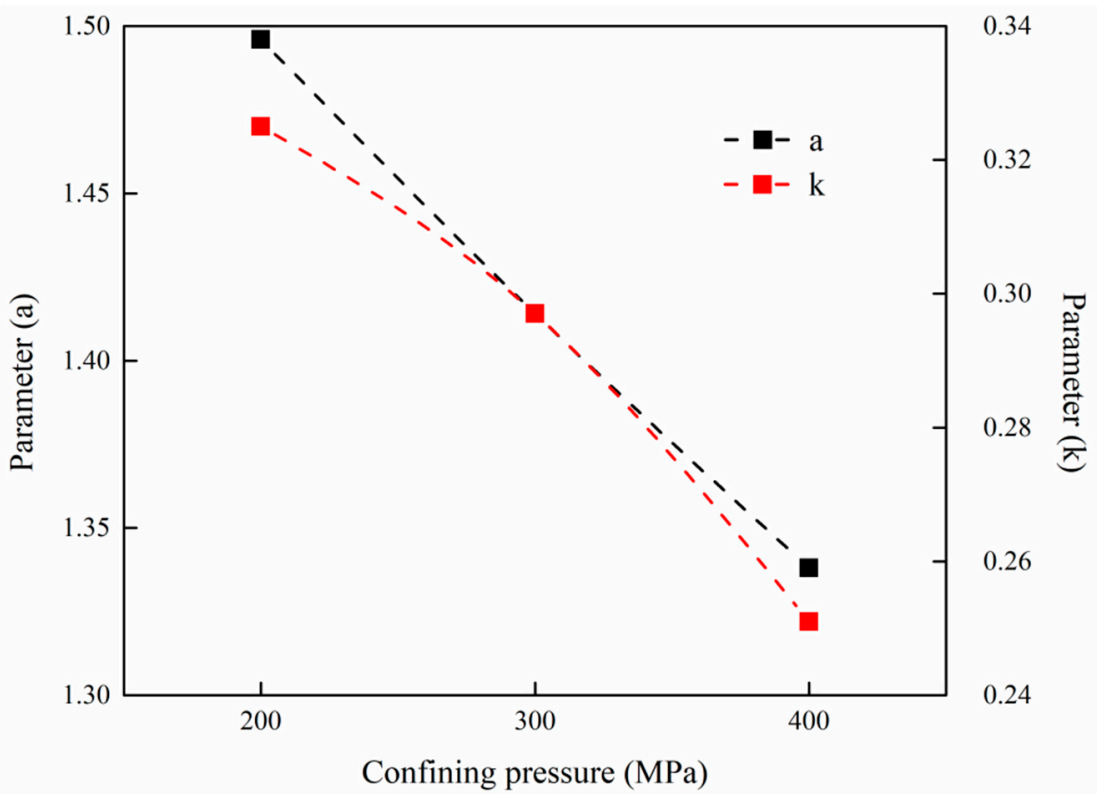

Figure 15. Parameter changes.

\section{Conclusions}

This article aims to study the problem of loess landslides caused by freeze-thaw cycles. Taking saturated undisturbed loess as the research object, the relationship between its strength and pore structure is analyzed. The changes of pore structure in loess were observed by scanning electron microscopy and nuclear magnetic resonance techniques, and analyzing the variation of porosity and shear strength. The following main conclusions were obtained:

(1) The freeze-thaw damage evolution of saturated undisturbed loess under freeze-thaw cycles was observed by scanning electron microscopy. It is found that the micro and small pores in the soil increase first and then decrease, and the medium and large pores reduce first and then increase. This is closely related to changes in the internal microstructure of the soil. The T2 spectrum distribution curve of saturated undisturbed loess under freeze-thaw cycles was obtained by using nuclear magnetic resonance technology. The pore distribution curve of loess under freeze-thaw cycles is obtained by equation inversion, which shows that the micro and small pores in the loess are gradually transitioning to the larger medium and large pores with the increase of the number of freeze-thaw cycles. It is reported that the freeze-thaw cycle is a dynamic process for the internal pore expansion of loess. The results obtained by NMR and SEM experiments confirm each other, and the pore distribution has similar changes.

(2) For saturated undisturbed loess, the freeze-thaw cycle breaks the original balance of the sample itself. During the freezing process, the soil particles are squeezed by the growth of ice crystals, the volume expands, and pores and fissures develop, forming a new soil skeleton structure. During the melting process, the melting of solid ice inside the rock sample cannot cause the complete restoration of the deformation of the soil skeleton particles. Therefore, during the freeze-thaw cycle, due to the effect of the frost-heaving force, the loess sample shows a decrease in strength and an increase in porosity.

(3) The strength of saturated undisturbed loess under freeze-thaw cycles is related to the change of porosity. The measurement of porosity is relatively simple. Therefore, consider establishing a functional relationship with the shear strength based on the change in porosity. According to the concept of freeze-thaw damage, an exponential function distribution between the two is derived. Fitting the variation of the porosity and the shear strength under different cycle times, the correlation between the two is good. Therefore, this research provides a new method for the 
non-destructive analysis of the strength of saturated loess by freezing and thawing, and a new idea for analyzing the loess landslide caused by the freezing and thawing cycle.

Author Contributions: Writing-Original Draft Preparation, Z.L.; Funding Acquisition, G.Y. and H.L. All authors have read and agreed to the published version of the manuscript.

Funding: This work has been supported by the National Natural Science Foundation of China (grant No. 51774231, 41702339), National Key Research and Development Program of China (grant No. 2018YFC0808705).

Acknowledgments: We thank the entire team for their efforts to improve the quality of the article. At the same time, we would like to thank editor for his timely handling of the manuscripts.

Conflicts of Interest: The authors declare no conflict of interest.

\section{References}

1. Peng, J.; Wang, G.; Wang, Q.; Zhang, F. Shear wave velocity imaging of landslide debris deposited on an erodible bed and possible movement mechanism for a loess landslide in Jingyang, Xi'an, China. Landslides 2017, 14, 1503-1512. [CrossRef]

2. Xu, X.; Guo, W.-Z.; Liu, Y.; Ma, J.; Wang, W.; Zhang, H.; Gao, H. Landslides on the Loess Plateau of China: A latest statistics together with a close look. Nat. Hazards 2017, 86, 1393-1403. [CrossRef]

3. Zhang, F.; Kang, C.; Chan, D.; Zhang, X.; Pei, X.; Peng, J. A study of a flowslide with significant entrainment in loess areas in China. Earth Surf. Process. Landf. 2017, 42, 2295-2305. [CrossRef]

4. Peng, J.; Sun, X.; Wang, W.; Sun, G. Characteristics of land subsidence, earth fissures and related disaster chain effects with respect to urban hazards in Xi'an, China. Environ. Earth Sci. 2016, 75, 1190. [CrossRef]

5. Qi, J.; Vermeer, P.A.; Cheng, G. A review of the influence of freeze-thaw cycles on soil geotechnical properties. Permafr. Periglac. Process 2010, 17, 245-252. [CrossRef]

6. Xu, J.; Wang, Z.; Ren, J.; Wang, S.; Jin, L. Mechanism of slope failure in loess terrains during spring thawing. J. Mt. Sci. 2018, 15, 845-858. [CrossRef]

7. Li, G.; Ma, W.; Mu, Y.; Wang, F.; Fan, S.; Wu, Y. Effects of freeze-thaw cycle on engineering properties of loess used as road fills in seasonally frozen ground regions, North China. J. Mt. Sci. 2017, 14, 356-368. [CrossRef]

8. Peng, J.; Lan, H.-X.; Qian, H.; Li, R.; Li, Z.; Zhuang, J.; Li, X.; Liu, S. Scientific research framework of livable yellow river. J. Eng. Geol. 2020, 28, 189-201. [CrossRef]

9. Xu, J.; Ren, J.; Wang, Z.; Wang, S.; Yuan, J. Strength behaviors and meso-structural characters of loess after freeze-thaw. Cold Reg. Sci. Technol. 2018, 148, 104-120. [CrossRef]

10. Wang, S.; Lv, Q.; Baaj, H.; Li, X.; Zhao, Y. Volume change behaviour and microstructure of stabilized loess under cyclic freeze-thaw conditions. Can. J. Civ. Eng. 2016, 43, 865-874. [CrossRef]

11. Li, G.; Wang, F.; Ma, W.; Fortier, R.; Mu, Y.; Mao, Y.; Hou, X. Variations in strength and deformation of compacted loess exposed to wetting-drying and freeze-thaw cycles. Cold Reg. Sci. Technol. 2018, 151, 159-167. [CrossRef]

12. Li, J.; Zhou, K.; Liu, W.; Deng, H. NMR research on deterioration characteristics of microscopic structure of sandstones in freeze-thaw cycles. Trans. Non-Ferrous Metals Soc. Ch. 2016, 26, 2997-3003. [CrossRef]

13. Tian, H.; Wei, C.; Wei, H.; Zhou, J. Freezing and thawing characteristics of frozen soils: Bound water content and hysteresis phenomenon. Cold Reg. Sci. Technol. 2014, 103, 74-81. [CrossRef]

14. Lu, Z.; Xian, S.; Yao, H.; Fang, R.; She, J. Influence of freeze-thaw cycles in the presence of a supplementary water supply on mechanical properties of compacted soil. Cold Reg. Sci. Technol. 2019, 157, 42-52. [CrossRef]

15. Yan, C.-G.; Zhang, Z.-Q.; Jing, Y.-L. Characteristics of strength and pore distribution of lime-flyash loess under freeze-thaw cycles and dry-wet cycles. Arab. J. Geosci. 2017, 10, 544. [CrossRef]

16. Xu, J.; Wang, Z.; Ren, J.; Yuan, J. Mechanism of shear strength deterioration of loess during freeze-thaw cycling. Geomech. Eng. 2018, 14, 307-314. [CrossRef]

17. Guo, L.; Shi, M.; Song, N.; Wan, Z.; Liu, H.; Liu, L. Anchorage of curcumin onto PVP enhances anti-tumor effect of curcumin. Med. Chem. Res. 2019, 28, 646-656. [CrossRef]

18. Yang, Y.; Li, W.; Ren, Y.; Cai, X. Preparation and thermal performance of novel form-stable phase change materials based on polyethylene glycol (PEG)/hollow glass microsphere composites for thermal energy storage. Polym. Bull. 2019, 76, 2711-2724. [CrossRef] 
19. Chen, Y.; Lin, S.; Wang, J.; Hsu, S.; Ma, C.C.M. Preparation and properties of graphene/carbon nanotube hybrid reinforced mortar composites. Mag. Concr. Res. 2019, 71, 395-407. [CrossRef]

20. Bakhshipour, Z.; Asadi, A.; Huat, B.; Sridharan, A.; Kawasaki, S. Effect of acid rain on geotechnical properties of residual soils. Soils Found 2016, 56, 1008-1020. [CrossRef]

21. Khodayar, A.; Nejati, H.-R. Effect of thermal-induced microcracks on the failure mechanism of rock specimens. Comput. Concr. 2018, 22, 93-100. [CrossRef]

22. Yang, X.; Jiang, A.; Li, M. Experimental investigation of the time-dependent behavior of quartz sandstone and quartzite under the combined effects of chemical erosion and freeze-thaw cycles. Cold Reg. Sci. Technol. 2019, 161, 51-62. [CrossRef]

23. Cardoso, R.; Ribeiro, D.; Neri, R. Bonding effect on the evolution with curing time of compressive and tensile strength of sand-cement mixtures. Soils Found 2018, 57, 655-668. [CrossRef]

24. Mousavi, S. Stabilization of compacted clay with cement and/or lime containing peat ash. Road Mater. Pavement Des. 2016, 18, 1304-1321. [CrossRef]

25. Lin, T.; Zhang, Y.; Yi, X.; Fan, T.; Wan, L. Time-frequency peak filtering for random noise attenuation of magnetic resonance sounding signal. Geophys. J. Int. 2018, 213, 727-738. [CrossRef]

26. Lin, T.; Yang, Y.; Teng, F.; Muller-Petke, M. Enabling surface nuclear magnetic resonance at high-noise environments using a pre-polarization pulse. Geophys. J. Int. 2018, 212, 1463-1467. [CrossRef]

27. Shushakov, O.; Maryasov, A. Bloch-Siegert Effect in Magnetic-Resonance Sounding. Appl. Magn. Reason. 2016, 47, 1021-1032. [CrossRef]

28. Coates, G.; Marschall, D.; Mardon, D.; Galford, J. A new characterization of bulk-volume irreducible using magnetic resonance. Log. Anal. 1997, 39, 51-63.

29. Lu, Y.; Liu, S.; Alonso, E.; Wang, L.; Xu, L.; Li, Z. Volume changes and mechanical degradation of a compacted expansive soil under freeze-thaw cycles. Cold Reg. Sci. Technol. 2019, 157, 206-214. [CrossRef]

30. Lemaitre, J. How to use damage mechanics. Nucl. Eng. Design 1987, 80, 233-245. [CrossRef]

31. Jia, H.; Xiang, W.; Shen, Y.; Yang., G. Discussion of the key issues within calculation of the fatigue damage of rockssubjected to freeze-thaw cycles. Ch. J. Rock Mech. Eng. 2017, 36, 335-346. [CrossRef]

Publisher's Note: MDPI stays neutral with regard to jurisdictional claims in published maps and institutional affiliations.

(C) 2020 by the authors. Licensee MDPI, Basel, Switzerland. This article is an open access article distributed under the terms and conditions of the Creative Commons Attribution (CC BY) license (http://creativecommons.org/licenses/by/4.0/). 